\title{
The Impact of Comorbidities on the Outcomes of Egyptian COVID-19 Patients: A Follow-Up Study
}

\author{
Reda M. Albadawy $D^{D},{ }^{1}$ Bismeen A. Jadoon ${ }^{D},{ }^{2}$ Mysara M. Mogahed $\left(\mathbb{D},{ }^{3}\right.$ \\ Mohamed E. Ibrahim (D), ${ }^{3}$ Tarek S. Essawy ${ }^{1},{ }^{4}$ Ahmed M. A. Amin $\left(\mathbb{D},{ }^{1}\right.$ \\ Marwa S. Abd-Elraouf $\left(\mathbb{D},{ }^{5}\right.$ and Mona A. Elawady ${ }^{5}{ }^{5}$ \\ ${ }^{1}$ Department of Hepatology Gastroenterology and Infectious Diseases, Benha University, Faculty of Medicine, Banha, Egypt \\ ${ }^{2}$ WHO Consultant, Research Coordinator at ERC-RCOG, Giza, Egypt \\ ${ }^{3}$ Internal Medicine Department, Benha University, Faculty of Medicine, Banha, Egypt \\ ${ }^{4}$ Chest Department, Benha University, Faculty of Medicine, Banha, Egypt \\ ${ }^{5}$ Public Health Department, Faculty of Medicine, Benha University, Banha, Egypt
}

Correspondence should be addressed to Mona A. Elawady; dr_monaelawady@yahoo.com

Received 27 December 2020; Revised 26 March 2021; Accepted 10 June 2021; Published 19 June 2021

Academic Editor: Felix Broecker

Copyright $\odot 2021$ Reda M. Albadawy et al. This is an open access article distributed under the Creative Commons Attribution License, which permits unrestricted use, distribution, and reproduction in any medium, provided the original work is properly cited.

\begin{abstract}
Objectives. This study evaluated the clinical manifestation of COVID-19 and adverse outcomes in patients with comorbidities (outcome: death). Methods. A comparative follow-up investigation involving 148 confirmed cases of COVID-19 was performed for a month (between April and May 2020) at Qaha Hospital to describe the clinical characteristics and outcomes resulting from comorbidities. Participants were divided into two clusters based on the presence of comorbidities. Group I comprised cases with comorbidities, and Group II included subjects without comorbidity. Survival distributions were outlined for the group with comorbidities after the follow-up period. Results. Fever (74.3\%), headache (78.4\%), cough (78.4\%), sore throat (78.4\%), fatigue (78.4\%), and shortness of breath (86.5\%) were the most prevalent symptoms observed in COVID-19 patients with comorbidities. Such patients also suffered from acute respiratory distress syndrome (37.8\%) and pneumonia three times more than patients without comorbidities. The survival distributions were statistically significant (chi-square $=26.06, p \leq 0.001$ ). Conclusion. Multiple comorbidities in COVID-19 patients are linked to severe clinical symptoms, disease complications, and critical disease progression. The presence of one or more comorbidities worsened the survival rate of patients.
\end{abstract}

\section{Introduction}

The Coronaviridae family has become the foremost microorganism triggering increasing disease outbreaks. It is a vast family of single-stranded RNA viruses (+ssRNA) that can be isolated from totally different animal species. These viruses can cross interspecies barriers and cause ill health in humans ranging from common cold to more severe respiratory diseases [1].

An ongoing public health emergency of international concern occurred due to an epidemic of COVID-19, which began in China in December 2019. Patients confirmed to have contracted this illness suffer outcomes ranging from mild to severe health issues to death. The incubation period of the disease is around 2-14 days after exposure. One or more subsequent symptoms could include (but not be restricted to) fever, cough, diarrhea, or labored breathing [2]. In January 2020, SARS-CoV-2 was believed to cause an outbreak of severe pneumonia; however, pneumonia is now well known to be a complication caused by COVID-19 [3]. COVID-19 has advanced with terrible speed since its discovery. The World Health Organization (WHO) declared a pandemic on 11 March 2020 [4]. By 15 April, more than $1,900,000$ cases and 123,000 deaths had been assumed worldwide [5].

The clinical spectrum of COVID-19 spans asymptomatic or mild symptomatic as well as clinical conditions characterized by respiratory failure. Artificial breathing aids and 
life support in intensive care units (ICU) are mandated for multiorgan and systemic manifestations such as sepsis, septic shock, and multiple organ dysfunction syndromes (MODS) [6].

The Chinese Center for Disease Control and Prevention (CCDC) has classified the clinical manifestations of the disease according to severity: delicate, severe, and critical. Delicate patients do not contract pneumonia. Severe patients display dyspnea, respiratory rate $\geq 30 \mathrm{~min}$, blood gas saturation $\left(\mathrm{SpO}_{2}\right) \leq 93 \%$, lung infiltrates $>50 \%$ within $24-48$ hours and/or $<300, \mathrm{PaO}_{2} / \mathrm{FiO}_{2}$ ratio, or $\mathrm{P} / \mathrm{F}$. This last measure denotes the ratio between the forces per unit area of the gas, $\mathrm{PaO}_{2}$, and therefore indicates the proportion of gas provided (fraction of impressed gas, $\mathrm{FiO}_{2}$ ). Critical patients suffer respiratory failure, septic shock, and/or MODS [7]. Acute respiratory distress syndrome (ARDS) implicates a significant new-onset respiratory failure or worsening of the related and already identified respiratory image. Distinct varieties of ARDS are distinguished based on degrees of hypoxia [8].

The number of patients currently diagnosed with COVID-19 has multiplied dramatically; however, the connection between comorbidity and patients with COVID-19 remains unclear. Previously conducted studies have demonstrated that comorbidities could lead to a poor prognosis. Identifying the major risk groups is crucial to decisionmaking concerning anti-COVID-19 therapy. To date, only a few systematic reviews have comprehensively explored whether the presence of common comorbidities increases risks for COVID-19 patients risk or better guides clinical practices. However, multiple studies and systematic reviews have reported the link between single risk factors and the severity of COVID-19 [9].

This study aimed to describe the clinical characteristics and outcomes observed in patients diagnosed with COVID19 in addition to comorbidities.

\section{Methods}

The research question probed whether comorbidities influenced the clinical characteristics and outcomes of COVID-19 patients.

The null hypothesis for the study read that COVID-19 patients with one or more comorbidity display clinical characteristics and outcomes identical to COVID-19 patients without comorbidities.

The alternative hypothesis for the study was COVID-19 patients with one or more comorbidity display different characteristics and outcomes from COVID-19 patients without comorbidities.

2.1. Study Design. A comparative follow-up study of one month was initiated from the time of diagnosis and was conducted between April and May 2020. One month is the mean period of hospital stay estimated by a pilot study performed on 37 COVID-19 patients (20\% of the calculated sample size). The results of the pilot trial were not included in the outcomes of the present study.
2.2. Sampling Technique. Random cluster sampling was deployed to select Qaha Central Hospital from all quarantine hospitals for COVID-19 patients in Egypt.

2.3. Sample Size Calculation. This study utilized results obtained from Open Epi, version 3, open-source calculator-SSCC, for the data extracted from another Egyptian study [10]. A minimal sample size of 180 was indicated.

The study population included 185 patients with COVID-19 who fulfill the specified inclusion criteria before adjusting for age and sex, after which the tally came to 148 .

A trained team of physicians accomplished the data collection from April to May 2020 according to the hospital's standard protocols.

2.4. The Inclusion Criteria. Stipulated for the study population, specified cases of COVID-19 were confirmed according to the real-time reverse transcriptase polymerase chain reaction (RT-PCR) test taken through nasal swabs. The patients were required to display one or more symptoms including fever, headache, cough, sore throat, sputum production, fatigue, shortness of breath (SOB), nausea, vomiting, diarrhea, nasal congestion, conjunctival congestion, myalgia, and arthralgia.

The patients were classified into two groups for comparison. Group I included COVID-19 patients diagnosed with one or more comorbidity including hypertension (HTN), coronary heart disease (CHD), cerebrovascular disease, diabetes (DM), chronic obstructive airway disease (COPD), chronic liver disease (CLD), chronic kidney diseases (CKD), and malignancy. Group II encompassed confirmed patients of COVID-19 without comorbidities. Patients in each group were further categorized into three subgroups according to age: $<18 \mathrm{y}, 18 \leq 60$, and $\geq 60 \mathrm{y}$. The primary measured outcomes included death or survival. Descriptive analysis was performed for factors such as demographic characteristics (age and gender). Demographic and clinical predictors were examined. Unadjusted relationships between both groups were determined. The results were later adjusted for age and gender through propensity score matching using IBM SPSS version 26 software (SPSS Inc., Chicago, ILL Company). Categorical data were presented as numbers and percentages and were analyzed using chi-square and Fisher's exact test, while quantitative data were expressed as mean \pm standard deviation and evaluated using ANOVA (F-test). Kaplan-Meier was employed for survival analysis. All tests were 2-sided, and a $p$ value of less than 0.05 was considered statistically significant.

\section{Results}

3.1. Presence of Comorbidity. Table 1 shows the prevalence of symptoms and the rate of complications experienced by patients classified into the two groups. Fever (74.3\%), headache, cough, sore throat, fatigue (equally presented as $78.4 \%)$, and SOB (86.5\%) were observed in patients 
TABLE 1: Distribution of the studied groups according to comorbidities.

\begin{tabular}{|c|c|c|c|c|c|}
\hline & \multicolumn{4}{|c|}{ Presence of comorbidity } & \multirow{3}{*}{$P$ value } \\
\hline & \multicolumn{2}{|c|}{$\begin{array}{l}\text { Group I (with } \\
\text { comorbidity) (74) }\end{array}$} & \multicolumn{2}{|c|}{$\begin{array}{l}\text { Group II (without } \\
\text { comorbidity) (74) }\end{array}$} & \\
\hline & $N$ & $(\%)$ & $N$ & $(\%)$ & \\
\hline \multicolumn{6}{|l|}{ Symptoms } \\
\hline History of fever & 55 & 74.3 & 21 & 28.4 & $<0.001^{* *}$ \\
\hline Conjunctival congestion & 20 & 27.0 & 2 & 2.7 & $<0.001^{* *}$ \\
\hline Nasal congestion & 24 & 32.4 & 16 & 21.6 & 0.14 \\
\hline Headache & 58 & 78.4 & 31 & 41.9 & $<0.001^{* *}$ \\
\hline Cough & 58 & 78.4 & 46 & 62.2 & $0.031^{*}$ \\
\hline Sore throat & 58 & 78.4 & 43 & 58.1 & $0.008^{* *}$ \\
\hline Sputum production & 4 & 5.4 & 1 & 1.4 & 0.37 \\
\hline Fatigue & 58 & 78.4 & 30 & 40.5 & $<0.001^{* *}$ \\
\hline Shortness of breath & 64 & 86.5 & 11 & 14.9 & $<0.001^{* *}$ \\
\hline Nausea & 22 & 29.7 & 4 & 5.4 & $<0.001^{* *}$ \\
\hline Vomiting & 12 & 16.2 & 2 & 2.7 & $0.005^{* *}$ \\
\hline Diarrhea & 4 & 5.4 & 1 & 1.4 & 0.37 \\
\hline Myalgia & 28 & 37.8 & 4 & 5.4 & $<0.001^{* * *}$ \\
\hline Cyanosis & 4 & 5.4 & 0 & 0.0 & 0.12 \\
\hline \multicolumn{6}{|l|}{ Complications } \\
\hline Pneumonia & 20 & 27.0 & 5 & 6.8 & $0.001^{* *}$ \\
\hline ARDS & 28 & 37.8 & 0 & 0.0 & $<0.001^{* * *}$ \\
\hline \multicolumn{6}{|l|}{ Outcome of hospitalization } \\
\hline Died & 28 & 37.8 & 0 & 0.0 & $<0.001^{* *}$ \\
\hline Survived & 46 & 62.2 & 74 & 100 & \\
\hline \multicolumn{6}{|l|}{ Gender } \\
\hline Male & 46 & 57.5 & 48 & 45.7 & $0.03^{*}$ \\
\hline Female & 34 & 42.5 & 67 & 54.3 & \\
\hline \multicolumn{6}{|l|}{ Age } \\
\hline$<18 \mathrm{y}$ & 0 & 0.0 & 12 & 11.4 & 0.002 \\
\hline$\geq 18 \mathrm{y}$ & 80 & 100 & 93 & 88.6 & \\
\hline \multicolumn{6}{|l|}{ Outcome of hospitalization } \\
\hline Died & 28 & 35.0 & 0 & 0.0 & $<0.001^{* *}$ \\
\hline Survived & 52 & 65.0 & 105 & 100 & \\
\hline After adjustment with gender & $N=(76)$ & & $N=(81)$ & & \\
\hline \multicolumn{6}{|l|}{ Gender } \\
\hline Male & 44 & 57.9 & 48 & 59.3 & 0.86 \\
\hline Female & 32 & 42.1 & 33 & 40.7 & \\
\hline \multicolumn{6}{|l|}{ Age } \\
\hline$<18 y$ & 0 & 0.0 & 12 & 14.8 & $<0.001^{* *}$ \\
\hline$\geq 18 \mathrm{y}$ & 76 & 100 & 69 & 85.2 & \\
\hline \multicolumn{6}{|l|}{ Outcome of hospitalization } \\
\hline Died & 28 & 36.8 & 0 & 0.0 & $<0.001^{* *}$ \\
\hline Survived & 48 & 63.2 & 81 & 100 & \\
\hline $\begin{array}{l}\text { After gender and age adjustment } \\
\text { Gender }\end{array}$ & $N=(74)$ & & $N=(74)$ & & \\
\hline Male & 42 & 56.8 & 43 & 58.1 & 0.87 \\
\hline Female & 32 & 43.2 & 31 & 41.9 & \\
\hline \multicolumn{6}{|l|}{ Age } \\
\hline$<18 y$ & 0 & 0.0 & 3 & 4.1 & 0.08 \\
\hline$\geq 18 \mathrm{y}$ & 74 & 100 & 71 & 95.9 & \\
\hline \multicolumn{6}{|l|}{ Outcome of hospitalization } \\
\hline Died & 28 & 37.8 & 0 & 0.0 & $<0.001^{* *}$ \\
\hline Survived & 46 & 62.2 & 74 & 100 & \\
\hline
\end{tabular}

${ }^{*}$ Significant at $p \leq 0.05 .{ }^{* *}$ Significant at $p<0.01$. Adjustment using propensity score matching. CT: computerized topography. 
TABLE 2: Distribution of the studied groups according to outcome of hospitalization.

\begin{tabular}{|c|c|c|c|c|c|}
\hline & & ne of 1 & over & & \\
\hline & & & & & $P$ value \\
\hline & $N$ & $(\%)$ & $N$ & $(\%)$ & \\
\hline Gender & & & & & \\
\hline Male & 16 & 57.1 & 69 & 57.5 & 0.97 \\
\hline Female & 12 & 42.9 & 51 & 42.5 & \\
\hline Age & & & & & \\
\hline$<18 y$ & 3 & 10.7 & 27 & 22.5 & 0.17 \\
\hline $18 \mathrm{y}$ & 12 & 42.9 & 57 & 47.5 & \\
\hline$\geq 60 y$ & 13 & 46.4 & 36 & 30.0 & \\
\hline Symptoms & & & & & \\
\hline History of fever & 11 & 39.3 & 81 & 67.5 & $0.006^{* *}$ \\
\hline Conjunctival congestion & 12 & 42.9 & 10 & 8.3 & $<0.001^{* *}$ \\
\hline Nasal congestion & 16 & 57.1 & 24 & 20.0 & $<0.001^{* *}$ \\
\hline Headache & 24 & 85.7 & 65 & 54.2 & $0.002^{* *}$ \\
\hline Cough & 28 & 100 & 76 & 63.3 & $<0.001^{* *}$ \\
\hline Sore throat & 28 & 100 & 73 & 60.8 & $<0.001^{* *}$ \\
\hline Sputum production & 0 & 0.0 & 5 & 4.2 & 0.58 \\
\hline Fatigue & 28 & 100 & 60 & 50.0 & $<0.001^{* *}$ \\
\hline Shortness of breath & 28 & 100 & 47 & 39.2 & $<0.001^{* *}$ \\
\hline Nausea & 8 & 28.6 & 18 & 15.0 & 0.089 \\
\hline Vomiting & 4 & 14.3 & 10 & 8.3 & 0.30 \\
\hline Diarrhea & 0 & 0.0 & 5 & 4.2 & 0.58 \\
\hline Myalgia & 16 & 57.1 & 16 & 13.3 & $<0.001^{* *}$ \\
\hline Cyanosis & 4 & 14.3 & 0 & 0.0 & $0.001^{* *}$ \\
\hline Complications & & & & & \\
\hline Pneumonia & 12 & 42.9 & 13 & 10.8 & $<0.001^{* *}$ \\
\hline ARDS & 28 & 100 & 0 & 0.0 & $<0.001^{* *}$ \\
\hline
\end{tabular}

*Significant at $p \leq 0.05 .{ }^{* *}$ Significant at $p<0.01$. CT: computerized topography.

designated to Group I, who also demonstrated statistically significant higher incidences of complications compared to those nominated to Group II.

3.2. Outcome of Hospitalization. Table 2 presents the outcomes including mortality, persistent hospitalized status, and discharge resulting from the hospitalization of COVID19 patients. The 30 -day mortality rate was higher for patients aged $\geq 60$ years $(57.1 \%)$ than those aged $18-60$ years of age. The mortality rate was significantly higher for COVID-19 patients with a history of comorbidities such as asthma, COPD, DM, HTN, CHD, CLD, and CKD (28.6\%, 14.3\%, $42.9 \%, 100 \%, 28.6 \%, 14.3 \%$, and $14.3 \%$, respectively). The same group of patients evinced significantly higher incidences of clinical symptoms such as fever, conjunctival congestion, nasal congestion, headache, cough, sore throat, fatigue, SOB, nausea, vomiting, myalgia, and cyanosis $(100 \%$, $42.9 \%, 57.1 \%, 85.7 \%, 100 \%, 100 \%, 100 \%, 100 \%, 28.6 \%$, $14.3 \%, 57.1 \%$, and $14.3 \%$, respectively).

The incidences of pneumonia (42.9\%) and ARDS (100\%) were significantly higher in patients with mortality outcomes on the $30^{\text {th }}$ day of admission $(p \leq 0.001)$. The same group exhibited a much greater incidence of ground glass appearance on CT scans in comparison with patients who remained hospitalized or were discharged at a later date (100\%) $(p \leq 0.001)$. The CT scan results of the latter group of patients revealed a positive ground glass appearance, multiple lobe involvement, and consolidations. The incidences of these CT scan observations among the three groups of patients (mortality, persistent hospitalized status, and discharge) were significant ( $p \leq 0.001,<0.001$, and 0.005 , respectively) and were computed at $100 \%, 71.4 \%$, and $57.1 \%$, respectively.

Multilinear logistic regression was performed to detect the predictors of mortality in COVID-19. It disclosed that the presence of one or more comorbidity was the preeminent cause of mortality. In order of predominance, the presence of comorbidities resulted in death (B (95\% CI): 36.27 (2.53-3.14)), cyanosis (23.18 (0.034-0.39)), and SOB (22.43 (9.55-13.64)) in patients designated to Group I (Table 3).

Figure 1 illustrates the results of a log-rank test run to determine whether there were differences in the survival distribution of the two groups. The survival distributions were statistically significant (chi-square $=26.06, p \leq 0.001$ ).

\section{Discussion}

A vulnerable population, with chronic health conditions such as diabetes and cardiovascular or lung disease, is at higher risk of developing severe illness with COVID-19. This population 
TABLE 3: Multilinear logistic regression to detect predictors that cause death to COVID-19 patients.

\begin{tabular}{lcccc}
\hline & $\mathrm{B}$ & $P$ value & Lower limit of 95\% CI & Upper limit of 95\% CI \\
\hline Comorbidity presence (Group II is reference) & 36.27 & $<0.001^{* *}$ & 2.53 & 3.14 \\
History of fever & -1.4 & 0.19 & -3.5 & 114.2 \\
Headache & -3.37 & 0.08 & 0.0 & 96.45 \\
Cough & -3.10 & 0.082 & -11.43 & 2.15 \\
SOB & 22.43 & $0.004^{* *}$ & 9.55 & 13.64 \\
Nasal congestion & 4.06 & 0.074 & 0.0 & 121.87 \\
Fatigue & 4.11 & 0.07 & -3.6 & 1.22 \\
Cyanosis & 23.18 & $0.001^{* *}$ & 0.034 & 0.39 \\
\hline
\end{tabular}

** Significant at $p<0.01$. CI: confidence interval.

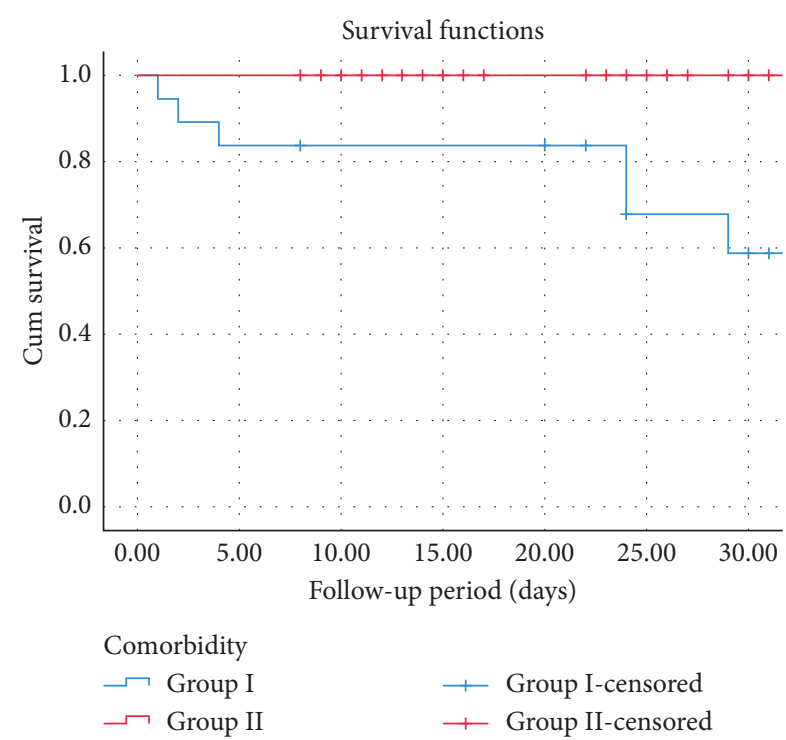

FIgure 1: Survival curve of COVID-19 patients according to comorbidity presence.

is also faced with an increased risk of death in the event of illness [11]. Patients with comorbidities recorded significantly ( $p=0.001$ ) higher percentages of complications, such as pneumonia and ARDS. This finding was congruent with the results reported by Bolin Wang et al., who revealed that patients with HTN (OR: 2.29, 95\% CI: 1.69-3.10, $p<0.001$ ), DM (OR: $2.47,95 \%$ CI: $1.67-3.66, p<0.001$ ), or COPD (OR: 5.97, 95\% CI: $2.49-14.29, p<0.001)$ were at increased risk of infections from COVID-19 [9].

Another study also reported the relationship between CHD and patients with severe COVID-19 [12]. Furthermore, five studies comprising 313 severe group cases and 1167 nonsevere group cases evaluated the role of CLD in patients with COVID-19. The meta-analysis divulged that the history of CLD did not increase the risk of disease progression (OR: 0.67, 95\% CI: 0.30-1.49, $p=0.326$ ) [13]. High blood pressure, dyslipidemia, diabetes, and obesity were marked as the main risk factors for poor prognoses because COVID-19 can increase blood glucose levels [14]. Some evidence indicates the arrhythmogenic potential of the drugs used in COVID-19 and moots the possibility that SARS-CoV-2 infection and other coronaviruses could cause arrhythmias [15]. The impact of underlying asthma, other allergic diseases, and T2 inflammation is suspected vis-à-vis susceptibility to COVID-19 and adverse disease outcomes. The better these interactions are understood, the more can the most vulnerable people be protected, including patients in high-risk groups [16].

$\mathrm{Li}$ et al. found that the levels of CRP, serum ferritin, and ESR (inflammation indicators) increased in COVID-19 patients and were associated with the greater severity of the disease. The levels of these indicators were higher in the patients with CVD than in those without CVD. This result indicates that COVID-19 exerted an additional impact on cases with a history of CVD [17].

Table 1 presents that the prevalence of symptoms in the current study is similar to the findings of Lechien et al. who reported the most common symptoms as headache (70.3\%), nasal obstruction $(67.8 \%)$, cough $(63.2 \%)$, asthenia $(63.3 \%)$, myalgia (62.5\%), rhinorrhea (60.1\%), and sore throat (52.9\%). Fever was reported by $45.4 \%$ of the patients [18]. Another study testified that $82.4 \%$ of the patients suffered from cough, $64.8 \%$ from fever, and $38.5 \%$ from fatigue [19]. Fever was associated both with the development of ARDS and with better outcomes [20].

Dysregulated inflammation, compromised immune systems, and levels of ACE2 receptors in kidneys may explain disease severity and mortality-related outcomes in COVID-19 patients with a history of CKD. The mortality rate was found to be $53.33 \%$ and $17.65 \%$, respectively, in COVID-19 patients with CKD and liver diseases [21].

This study reported a 30 -day mortality rate of $17.8 \%$ due to COVID-19. This incidence is congruent with the assertion of a mortality rate of $13 \%$, made by another study [22]. Richardson et al. reported age to be a risk factor for COVID19. Age was also a factor that could help detect the outcomes of contracting COVID-19. Patients with DM or HTN who died and patients who had to be given intensive care ventilation or ICU care also belonged to older age groups [23].

4.1. Limitations of the Study. The current study admits to two limitations. First, only patients with relatively severe pneumonia due to COVID-19 were hospitalized during this period because of limited resources. Second, this study was conducted at a single hospital. The difference in population characteristics at various locations may produce different results.

\section{Conclusion}

In summary, the presence of multiple comorbidities in patients diagnosed with COVID-19 indicated significant 
symptoms and clinical manifestations and predicted a severe disease progression. The existence of more than two comorbidities worsened the survival rate of patients. A thorough assessment of comorbidities at the time of hospital admission may help establish the risk stratification of patients with COVID-19.

It is therefore suggested that a COVID-19 patient with one or more comorbidity may be saved if proper medical service is available. Prevention should be attempted by all possible means including vaccination and should be prioritized for high-risk populations and people with risk factors, especially chronic diseases. The management of a chronic ailment requires dietary modification, regular exercise, and adequate adherence to medications. The realities of isolation, social distancing, and lockdowns of cities pose many complex challenges for such individuals, significantly influencing their health and lifestyles.

\section{Data Availability}

The data used to support the findings of this study are available from the corresponding author upon request.

\section{Ethical Approval}

The research protocol was approved before the study was conducted by the Research Committee of Benha Faculty of Medicine (RC no.: 262020).

\section{Consent}

Written informed consent was obtained from all patients or their first-degree relatives.

\section{Conflicts of Interest}

The authors declare that they have no conflicts of interest.

\section{Acknowledgments}

The authors would like to thank all those who have supported this study, in any manner. The authors are especially grateful to the Qaha Hospital Manager and all the participants (patients) of the study.

\section{References}

[1] S. Perlman and J. Netland, "Coronaviruses post-SARS: update on replication and pathogenesis," Nature Reviews Microbiology, vol. 7, no. 6, pp. 439-450, 2009.

[2] N. Mustafa, "Plenareno infectious and chronic diseases conference," in Proceedings of the Barcelona International Terminal (BIT), vol. 6, Barcelona, Spain, 2020.

[3] P. Zhou, X.-L. Yang, X.-G. Wang et al., "A pneumonia outbreak associated with a new coronavirus of probable bat origin," Nature, vol. 579, no. 7798, pp. 270-273, 2020.

[4] World Health Organization, WHO Director-General's Opening Remarks at the Media Briefing on COVID-19, WHO, Geneva, Switzerland, 2020.

[5] World Health Organization, Coronavirus Disease 2019 (COVID-19), WHO, Geneva, Switzerland, 85, 2021.
[6] C. Huang, Y. Wang, X. Li et al., "Clinical features of patients infected with 2019 novel coronavirus in Wuhan, China," The Lancet, vol. 395, no. 10223, pp. 497-506, 2020.

[7] Z. Wu and J. M. McGoogan, "Characteristics of and important lessons from the coronavirus disease 2019 (COVID19) outbreak in China," JAMA, vol. 323, no. 13, pp. 1239-1242, 2020.

[8] A. Kogan, M. J. Segel, E. Ram et al., "Acute respiratory distress syndrome following cardiac surgery: comparison of the American-European consensus conference definition versus the berlin definition," Respiration, vol. 97, no. 6, pp. 518-524, 2019.

[9] A. Sanyaolu, C. Okorie, A. Marinkovic et al., "Comorbidity and its impact on patients with COVID-19," SN Comprehensive Clinical Medicine, vol. 2, no. 8, pp. 1069-1076, 2020.

[10] E. M. Khedr, E. Daef, A. M. Hussein et al., "Impact of comorbidities on COVID-19 outcome," MedRxiv preprint, 2020.

[11] M. Nishiga, D. W. Wang, Y. Han, D. B. Lewis, and J. C. Wu, "COVID-19 and cardiovascular disease: from basic mechanisms to clinical perspectives," Nature Reviews Cardiology, vol. 17, no. 9, pp. 543-558, 2020.

[12] A. V. Kulkarni, P. Kumar, H. V. Tevethia et al., "Systematic review with meta-analysis: liver manifestations and outcomes in COVID-19," Alimentary Pharmacology \& Therapeutics, vol. 52, no. 4, pp. 584-599, 2020.

[13] S. Lim, J. H. Bae, H.-S. Kwon, and M. A. Nauck, "COVID-19 and diabetes mellitus: from pathophysiology to clinical management," Nature Reviews Endocrinology, vol. 17, no. 1, pp. 11-30, 2020.

[14] J. A. San Román, A. Uribarri, I. J. Amat-Santos, Á. Aparisi, P. Catalá, and J. R. González-Juanatey, "The presence of heart disease worsens prognosis in patients with COVID-19," Revista Española de Cardiología (English Edition), vol. 73, no. 9, pp. 773-775, 2020.

[15] M. Morais-Almeida, H. Pité, R. Aguiar, I. Ansotegui, and J. Bousquet, "Asthma and the coronavirus disease 2019 pandemic: a literature review," International Archives of Allergy and Immunology, vol. 181, no. 9, pp. 680-688, 2020.

[16] P. Katulanda, H. A. Dissanayake, I. Ranathunga et al., "Prevention and management of COVID-19 among patients with diabetes: an appraisal of the literature," Diabetologia, vol. 63, no. 8, pp. 1440-1452, 2020.

[17] M. Li, Y. Dong, H. Wang et al., "Cardiovascular disease potentially contributes to the progression and poor prognosis of COVID-19," Nutrition, Metabolism and Cardiovascular Diseases, vol. 30, no. 7, pp. 1061-1067, 2020.

[18] J. R. Lechien, C. M. Chiesa-Estomba, S. Place et al., "Clinical and epidemiological characteristics of 1420 European patients with mild to moderate coronavirus disease 2019," Journal of Internal Medicine, vol. 288, no. 3, pp. 335-344, 2020.

[19] X.-Y. Zhao, X.-X. Xu, H.-S. Yin et al., "Clinical characteristics of patients with 2019 coronavirus disease in a non-Wuhan area of Hubei province, China: a retrospective study," $B M C$ Infectious Diseases, vol. 20, no. 1, p. 311, 2020.

[20] C. Wu, X. Chen, Y. Cai et al., "Risk factors associated with acute respiratory distress syndrome and death in patients with coronavirus disease 2019 pneumonia in Wuhan, China," JAMA Internal Medicine, vol. 180, no. 7, pp. 934-943, 2020.

[21] T. Oyelade, J. Alqahtani, and G. Canciani, "Prognosis of COVID-19 in patients with liver and kidney diseases: an early systematic review and meta-analysis," Tropical Medicine and Infectious Disease, vol. 5, no. 2, 2020. 
[22] N. M. Kuderer, T. K. Choueiri, D. P. Shah et al., "Clinical impact of COVID-19 on patients with cancer (CCC19): a cohort study," The Lancet, vol. 395, no. 10241, pp. 1907-1918, 2020.

[23] S. Richardson, J. S. Hirsch, M. Narasimhan et al., "Presenting characteristics, comorbidities, and outcomes among 5700 patients hospitalized with COVID-19 in the New York city area," JAMA, vol. 323, no. 20, p. 2052, 2020. 